\title{
Use of Inpatient Palliative Care Services in Patients With Advanced Cancer Receiving Critical Care Therapies
}

\author{
Kah Poh Loh, BMedSci, MBBCh BAOª Maya Abdallah, MD' ${ }^{\text {; }}$ Meng-Shiou Shieh, $\mathrm{PhD}^{c}$; \\ Mihaela S. Stefan, MD, PhD ${ }^{\mathrm{b}, \mathrm{c}}$; Penelope S. Pekow, PhD ${ }^{\mathrm{c}, \mathrm{d}}$; Peter K. Lindenauer, MD, MSc, MHM ${ }^{\mathrm{b}, \mathrm{c}}$; \\ Supriya G. Mohile, MD, MS ; Dilip Babu, MDª and Tara Lagu, MD, MPH',
}

\begin{abstract}
Background: Invasive mechanical ventilation (IMV), dialysis for acute kidney failure, and other critical care therapies (CCTs) are associated with a high risk for complications in patients with metastatic cancer. Inpatient palliative care (IPC) can assist in assessing patients' preferences for life-prolonging treatment at the end of life. This study investigated the use pattern of IPC, outcomes (in-hospital mortality, length of stay [LOS], discharge destination, and cost of care), and predictors of IPC use in patients with metastatic cancer who received CCTs. We hypothesized that IPC services are underused in this cohort. Methods: In this retrospective cohort study, we used the 2010 California State Inpatient Databases to identify adults with metastatic cancer who received CCTs that are common and reliably coded (IMV, tracheostomy, percutaneous endoscopic gastrostomy tube, dialysis for acute kidney failure, and total parenteral nutrition). We determined IPC use in all patients, in those who received IMV, and across 4 cancer subtypes (lung, breast, colorectal, and genitourinary). Outcomes were assessed based on IPC use. Multivariable analyses were used to investigate factors associated with IPC use. Results: We identified 5,862 hospitalizations, $19.8 \%$ of which used IPC services. IPC use varied across cancer subtypes (lung, 28.3\%; breast, $22.4 \%$; colorectal, $12.8 \%$; genitourinary, $16.1 \% ; P<.01)$. Patients who received and did not receive IPC services had high in-hospital mortality rates $(63.9 \%$ and $29.8 \%$, respectively), and costs of care and LOS were lower in survivors who received IPC compared with those who did not. Predictors of IPC use were lung cancer (vs colorectal or genitourinary cancer), higher comorbidity score, do-not-resuscitate status on admission or within 24 hours of admission, infections (vs cancer-related diagnoses), and higher hospital bed count. Conclusions: Use of IPC was low in the cohort who received CCTs with poor outcomes, although data on outpatient palliative care services is lacking. Predictors of IPC use may be used to identify patients who may benefit from these services.
\end{abstract}

J Natl Compr Canc Netw 2018;16(9):1055-1064 doi: 10.6004/jnccn.2018.7039

Cancer mortality rates have been declining over the past 2 decades, and the observed improvements in survival are partially attributable to advances in treatment options. ${ }^{1,2}$ Treatment-associated complications and disease progression have led to an increase in emergency

aDivision of Hematology/Oncology, James P. Wilmot Cancer Institute, University of Rochester/Strong Memorial Hospital, Rochester, New York; ${ }^{b}$ Department of Internal Medicine, and Institute for Healthcare Delivery and Population Science, University of Massachusetts Medical SchoolBaystate, Springfield, Massachusetts; and dDepartment of Biostatistics and Epidemiology, University of Massachusetts, Amherst, Massachusetts.

Submitted December 8, 2017; accepted for publication April 26, 2018. The authors have disclosed that they have no financial interests, arrangements, affiliations, or commercial interests with the manufacturers of any products discussed in this article or their competitors.

Dr. Lagu is supported by the National Heart, Lung, and Blood Institute of the NIH (K01 HL114745). Dr. Lagu has received consulting fees from the Institute for Healthcare Improvement, under contract to CMS, for her work department visits, hospitalizations, and intensive care unit (ICU) admissions during the course of treatment. ${ }^{3}$ Critical care therapies (CCTs), such as invasive mechanical ventilation (IMV), can be lifesaving; however, the inherent risks (eg, infections, need for hospitaliza-

on a project to help health systems achieve disability competence. Dr. Stefan is supported by the National Heart, Lung, and Blood Institute of the National Institutes of Health (1 K01HL11463101A1). Dr. Lindenauer was supported by a grant from the National Heart, Lung, and Blood Institute (K24HL132008).

Author contributions: Study concept: Loh, Stefan, Lindenauer, Lagu. Data curation: Loh. Data analysis: Shieh, Pekow. Investigation: All authors. Methodology: Loh, Shieh, Stefan, Pekow, Lindenauer, Lagu. Supervision: Lagu. Manuscript preparation: Loh, Abdallah. Critical revision: Shieh, Pekow, Lindenauer, Mohile, Babu, Lagu.

Correspondence: Kah Poh Loh, BMedSci, MBBCh BAO, James P. Wilmot Cancer Institute, Strong Memorial Hospital, University of Rochester Medical Center, 601 Elmwood Avenue, Box 704, Rochester, NY 14642. Email: Melissalkp@gmail.com 
tions) are higher in patients with advanced cancer given its incurable nature and the frequent need for prolonged treatment., ${ }^{4,5}$

A prior systematic review showed that the integration of palliative care interventions in the care of patients with life-limiting illnesses in the outpatient, acute care, and ICU settings can reduce ICU admissions and length of stay (LOS). ${ }^{6}$ In addition, palliative care services have been shown to improve symptom management, quality of life, and survival in some patients with metastatic cancer, and may also help reduce healthcare costs. ${ }^{7-9}$ However, the use pattern of inpatient palliative care (IPC) services for patients with advanced cancer receiving CCTs has not been well characterized. In patients with metastatic head and neck cancer, use of IPC services was reported to be $5 \% .{ }^{10}$ In another study, IPC services were used in $17 \%$ of patients admitted to the oncology service. ${ }^{11}$ Overall, these rates were low considering the poor prognoses of patients with advanced cancer admitted to the hospital.

In this study of hospitalized patients with metastatic cancer who received CCTs, we investigated IPC use and described outcomes (including in-hospital mortality, LOS, discharge destination, and cost of care) of patients who received IPC services and those who did not, and considered predictors of IPC use. Results were assessed based on IPC use to illustrate the poor outcomes associated with this population. We also looked at the use pattern of IPC services across the 4 most common cancer subtypes in our population (lung, breast, colorectal, and genitourinary). We hypothesized that use of IPC services was low in this cohort of patients.

\section{Methods}

\section{Design, Data Source, and Subjects}

For this retrospective observational cohort study, data were obtained from the 2010 Healthcare Cost and Utilization Project (HCUP), California State Inpatient Databases (CA SID), created through a Federal-State-Industry partnership and sponsored by the Agency for Healthcare Research and Quality (AHRQ). The CA SID contains information on inpatient hospital discharge records from $>98 \%$ of community hospitals in California. This information includes principal and secondary diagnoses and procedures, admission and discharge status, patient de- mographics, expected payment source, total charges, do-not-resuscitate (DNR) status at time of or within 24 hours of hospital admission, and LOS. Because the CA SID contains deidentified information, the Baystate Medical Center Institutional Review Board determined that the project did not meet the criteria for human subjects research.

We included one random admission (using the VisitLink variable on CA SID, and admission was selected randomly using the RANUNI function and seed on SAS 9.3 [SAS Institute Inc.]) of patients aged $\geq 18$ years with metastatic cancer who received CCTs. One admission was selected to exclude patients admitted multiple times. In addition, random selection, instead of the first or last admission, was performed to ensure that patients had equal probability of experiencing the outcome (eg, mortality). CCTs were defined based on the following ICD-9. CM procedure codes: IMV, 96.70-96.72; percutaneous endoscopic gastrostomy (PEG) tube, 43.11; dialysis for acute kidney failure, both 584.5-585.9 and 39.95 to exclude patients on chronic dialysis; total parenteral nutrition (TPN), 99.15; and tracheostomy, 31.1, 31.21, 31.29. The ICD-9 codes to identify patients with metastatic cancer have been described previously. ${ }^{12,13}$ We studied the use of these specific CCTs because they are more commonly used, are measures that typically require consent from patients or their healthcare proxies and are therefore considered more invasive than others, and are reliably coded.

\section{Patient and Clinical Characteristics}

We collected demographics including age, sex, race, insurance provider, comorbidities (modified combined comorbidity score derived from the Elixhauser and Charlson comorbidity index), cancer subtype, DNR status, principal diagnosis, teaching hospital status, and hospital bed count. ${ }^{14}$ The CA SID determined that DNR status was present if it was written at or within 24 hours of hospital admission. We classified principal diagnosis using the Clinical Classifications Software, a tool developed by AHRQ that collapses ICD-9 codes. These diagnoses were further collapsed into various categories (cancer-related diagnoses, infections, other pulmonary disorders, other cardiovascular and circulation disorders, other neurologic disorders, other gastrointestinal disorders, fluid and electrolyte/renal disorders, and others) by 
the investigators. Teaching hospital status was determined after merging the American Hospital Association Annual Survey, and hospital bed count was classified as small $(<200)$, medium (200-399), or large $(\geq 400)$.

\section{Measures}

Use of IPC services was defined using the ICD-9 diagnosis code V66.7. ${ }^{15,16}$ Two prior validation studies showed that it has a sensitivity of $66.3 \%$ to $83 \%$ and specificity of $95 \%$ to $99.1 \% .{ }^{15,16}$ We assessed several outcomes, including inpatient mortality, LOS, discharge destination, and cost of care in those who did and did not receive IPC services. The CA SID contains hospital charges calculated by hospital accounting systems (excluded hospital-based physician fees) from the index hospitalization, and the HCUP hospital-specific cost-to-charge ratios were used to convert charges to costs. ${ }^{17}$

\section{Analyses}

Patient characteristics and outcomes were described using the following descriptive analyses: counts and percentages, means, medians, and standard deviations (SD). We also described characteristics and outcomes of patients who did and did not receive IPC services (chi-square tests for categorical variables and Wilcoxon rank sum tests for continuous variables). Given the higher mortality associated with IMV use, separate analyses were performed for the subgroup of patients who received this intervention. Use of IPC services and outcomes were also described within strata defined by 4 of the most common cancer subtypes (lung, breast, colorectal, and genitourinary). For these subgroups, we adjusted for multiple comparisons using a Bonferroni correction, and tests were considered significant if $P<.0125$. Multivariable logistic regressions with generalized estimating equations accounting for hospital clustering effect were used to examine factors (age, sex, race, insurance provider, comorbidities, cancer subtype, DNR status, principal diagnosis, teaching hospital status, and hospital bed count) associated with the use of IPC services in the CCT and IMV groups. All analyses were performed using SAS 9.4 (SAS Institute Inc.).

\section{Results}

\section{CCT Group Characteristics and Outcomes}

A total of 5,862 admissions involving the administration of CCTs were included (Table 1). Mean patient age was 66.4 years (SD, 13.5), 50.2\% were men, and $58.5 \%$ were white. Commonly used CCTs included IMV (51.8\%), TPN (37.5\%), PEG tube (14.1\%), tracheostomy $(8.4 \%)$, and dialysis for acute kidney failure $(8.1 \%)$. Among cancer diagnoses, lung was the most common $(14.1 \%)$, followed by colorectal (13.9\%) and genitourinary (7.6\%; prostate: $3.9 \%$, bladder: $1.8 \%$, kidney: $1.8 \%$, other: $0.1 \%$ ); Figure 1 shows the use of the various therapies across the cancer subtypes. DNR status at time of or within 24 hours of hospital admission was documented in $14.2 \%$ of patients. Approximately $30 \%$ of admissions were in hospitals with $\geq 400$ beds. In-hospital mortality was $36.5 \%$, mean LOS was 15.8 days (SD, $14.7), 66.4 \%$ of survivors were discharged to home, and mean cost of care among survivors was $\$ 51,397$ (SD, \$44,684).

\section{Use of IPC Services}

In the full cohort of patients hospitalized with metastatic cancer who received CCTs, $19.8 \%$ received IPC services (Figure 2) compared with $23.4 \%$ of patients in the IMV subgroup.

\section{Characteristics and Outcomes of the CCT Group by IPC Use}

No significant demographic differences were noted in patients who received IPC services compared with those who did not (Table 1). Compared with patients who did not use IPC services, those who did were more likely to have lung cancer $(20.2 \%$ vs $12.6 \% ; P<.01)$, higher mean comorbidity scores $(2.1$ vs $2.0 ; P<.01)$, and documented DNR status at time of admission or within 24 hours $(24.7 \%$ vs $11.7 \%$; $P<.01)$, and to be admitted to hospitals with higher bed count ( $\geq 400$ beds: $32.9 \%$ vs $29.4 \%$; $P<.01$ ). Inhospital mortality was high in both those who received IPC services and those who did not (63.9\% vs $29.8 \% ; P<.01)$. Among survivors, LOS (14.3 vs 16.0 days; $P=.01)$ and cost of care $(\$ 42,775$ vs $\$ 52,387$; $P<.01)$ were lower in those who received IPC services. Discharge destinations were not statistically significant between the 2 groups. 
Loh et al

Table 1. Outcomes and Demographic and Clinical Characteristics of Patients on CCTs

\begin{tabular}{|c|c|c|c|c|}
\hline Characteristics & $\begin{array}{l}\text { All Admissions } \\
(\mathrm{N}=5,862)\end{array}$ & $\begin{array}{c}\text { IPC } \\
(\mathrm{N}=1,158)\end{array}$ & $\begin{array}{c}\text { No IPC } \\
(\mathrm{N}=4,704)\end{array}$ & $P$ Value \\
\hline Age, n (\%) & & & & .87 \\
\hline$<55 y$ & $1,073(18.3 \%)$ & $210(18.1 \%)$ & $863(18.3 \%)$ & \\
\hline $55-64 y$ & $1,400(23.9 \%)$ & $287(24.8 \%)$ & $1,113(23.7 \%)$ & \\
\hline $65-74 y$ & $1,605(27.4 \%)$ & $310(26.8 \%)$ & $1,295(27.5 \%)$ & \\
\hline$\geq 75 y$ & $1,784(30.4 \%)$ & $351(30.3 \%)$ & $1,433(30.5 \%)$ & \\
\hline Male sex, n (\%) & $2,944(50.2 \%)$ & $573(49.5 \%)$ & $2,371(50.4 \%)$ & .73 \\
\hline Race, n (\%) & & & & .18 \\
\hline White & $3,432(58.5 \%)$ & $692(59.8 \%)$ & $2,740(58.2 \%)$ & \\
\hline Black & $536(9.1 \%)$ & $106(9.1 \%)$ & $430(9.1 \%)$ & \\
\hline Hispanic & $928(15.8 \%)$ & $173(14.9 \%)$ & $755(16.1 \%)$ & \\
\hline Other $^{\mathrm{b}}$ & $728(12.4 \%)$ & $153(13.2 \%)$ & $575(12.2 \%)$ & \\
\hline Missing & $238(4.1 \%)$ & $34(2.9 \%)$ & $204(4.3 \%)$ & \\
\hline Insurance provider, n (\%) & & & & .26 \\
\hline Medicare & $3,282(56.0 \%)$ & $648(56.0 \%)$ & $2,634(56.0 \%)$ & \\
\hline Medicaid & $742(12.7 \%)$ & $135(11.7 \%)$ & $607(12.9 \%)$ & \\
\hline Private & $1,647(28.1 \%)$ & $336(29.0 \%)$ & $1,311(27.9 \%)$ & \\
\hline No charge/other & $87(1.5 \%)$ & $23(2.0 \%)$ & $64(1.4 \%)$ & \\
\hline Self-pay & $104(1.8 \%)$ & $16(1.4 \%)$ & $88(1.9 \%)$ & \\
\hline Mean comorbidity score (SD) & $2.0(2.0)$ & $2.1(1.8)$ & $2.0(2.0)$ & $<.01$ \\
\hline DNR, n (\%) & & & & $<.01$ \\
\hline Yes & $835(14.2 \%)$ & $286(24.7 \%)$ & $549(11.7 \%)$ & \\
\hline No & $5,025(85.7 \%)$ & $872(75.3 \%)$ & $4,153(88.3 \%)$ & \\
\hline Missing & $2(0.1 \%)$ & $0(0 \%)$ & $2(0 \%)$ & \\
\hline Cancer subtype, n (\%) & & & & $<.01$ \\
\hline Lung & $826(14.1 \%)$ & $234(20.2 \%)$ & $592(12.6 \%)$ & \\
\hline Breast & $268(4.6 \%)$ & $60(5.2 \%)$ & $208(4.4 \%)$ & \\
\hline Colorectal & $813(13.9 \%)$ & $104(9.0 \%)$ & $709(15.1 \%)$ & \\
\hline Genitourinary & $448(7.6 \%)$ & $72(6.2 \%)$ & $376(8.0 \%)$ & \\
\hline Other cancer or $>1$ subtype & $2,163(36.9 \%)$ & $402(34.7 \%)$ & $1,761(37.4 \%)$ & \\
\hline Not identified & $1,344(22.9 \%)$ & $286(24.7 \%)$ & $1,058(22.5 \%)$ & \\
\hline Principal diagnosis, n (\%) & & & & $<.01$ \\
\hline Cancer-related disorders & $2,841(48.5 \%)$ & $514(44.4 \%)$ & $2,327(49.5 \%)$ & \\
\hline Infections & $1,135(19.4 \%)$ & $277(23.9 \%)$ & $858(18.2 \%)$ & \\
\hline Other pulmonary disorders & $511(8.7 \%)$ & $122(10.5 \%)$ & $389(8.3 \%)$ & \\
\hline Other cardiovascular and circulation disorders & $239(4.1 \%)$ & $47(4.1 \%)$ & $192(4.1 \%)$ & \\
\hline Other neurologic disorders & $72(1.2 \%)$ & $15(1.3 \%)$ & $57(1.2 \%)$ & \\
\hline Other gastrointestinal disorders & $512(8.7 \%)$ & $91(7.9 \%)$ & $421(9.0 \%)$ & \\
\hline Fluid and electrolyte/renal disorders & $209(3.6 \%)$ & $41(3.5 \%)$ & $168(3.6 \%)$ & \\
\hline Others & $343(5.9 \%)$ & $51(4.4 \%)$ & $292(6.2 \%)$ & \\
\hline Teaching hospital, $\mathrm{n}(\%)^{c}$ & $2,696(46.0 \%)$ & $513(44.3 \%)$ & $2,183(46.4 \%)$ & .02 \\
\hline Hospital bed count, n (\%) & & & & $<.01$ \\
\hline Small $(<200)$ & $1,511(25.8 \%)$ & $228(19.7 \%)$ & $1,283(27.3 \%)$ & \\
\hline Medium (200-399) & $2,520(43.0 \%)$ & $527(45.5 \%)$ & $1,993(42.4 \%)$ & \\
\hline Large $(\geq 400)$ & $1,763(30.1 \%)$ & $381(32.9 \%)$ & $1,382(29.4 \%)$ & \\
\hline Missing & $68(1.2 \%)$ & $22(1.9 \%)$ & $46(1.0 \%)$ & \\
\hline In-hospital mortality, n (\%) & $2,140(36.5 \%)$ & $740(63.9 \%)$ & $1,400(29.8 \%)$ & $<.01$ \\
\hline Discharge among survivors, $\mathrm{n}(\%)$ & & & & .07 \\
\hline Home, home healthcare & $2,473(66.4 \%)$ & $282(67.5 \%)$ & $2,191(66.3 \%)$ & \\
\hline SNF, ICF, or facility & $970(26.1 \%)$ & $95(22.7 \%)$ & $875(26.5 \%)$ & \\
\hline Short-term hospital, AMA, or other & $279(7.5 \%)$ & $41(9.8 \%)$ & $238(7.2 \%)$ & \\
\hline Mean cost of care among survivors (SD) ${ }^{d}$ & $\$ 51,397(\$ 44,684)$ & $\$ 42,775(\$ 40,136)$ & $\$ 52,387(\$ 45,078)$ & $<.01$ \\
\hline Mean length of stay among survivors (SD), $\mathrm{d}^{\mathrm{a}}$ & $15.8(14.7)$ & $14.3(13.3)$ & $16.0(14.9)$ & .01 \\
\hline
\end{tabular}

Abbreviations: AMA, against medical advice; CCTs, critical care therapies; DNR, do not resuscitate; ICF, intermediate care facility; IPC, inpatient palliative care services; SNF, skilled nursing facility.

a8 missing.

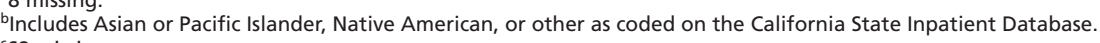

'68 missing.

d584 missing.

\section{Characteristics and Outcomes of the IMV Subgroup by IPC Use}

In patients receiving IMV, those who used IPC services were more likely to be white $(59.7 \%$ vs 54.4\%; $P=.05)$, have documented DNR status at time of admission or within 24 hours $(23.6 \%$ vs
$13.1 \% ; P<.01)$, and to be admitted to hospitals with higher bed count ( $\geq 400$ beds: $35.5 \%$ vs $28.3 \%$; $P<.01$ ) compared with those who did not receive IPC services (Table 2 ). In-hospital mortality in patients who received and did not receive IPC services was $82.0 \%$ and $49.7 \%$, respectively. Among survivors, LOS, dis- 


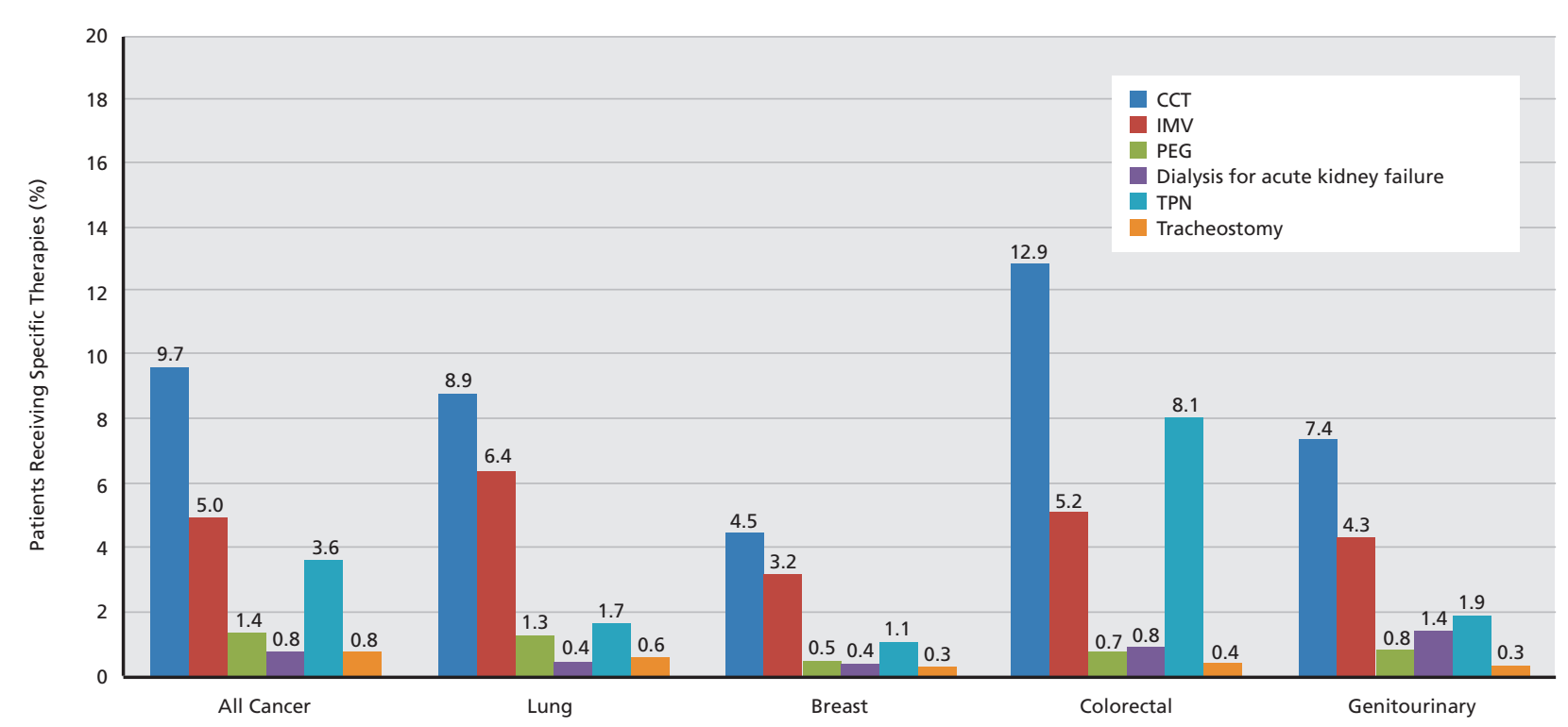

Figure 1. Comparison of various CCTs across cancer subtypes (all $P<.01$ ). The denominator is the number of admissions with metastatic cancer ( $\mathrm{n}=60,507)$. Abbreviations: CCT, critical care therapies; IMV, invasive mechanical ventilation; PEG, percutaneous endoscopic gastrostomy; TPN, total parenteral nutrition.

charge destination, and cost of care were not statistically significant between the 2 groups.

\section{Use of IPC Services Across Cancer Subtypes}

In the CCT group, patients who received IPC services differed slightly across cancer subtypes (lung, 28.3\%; breast, $22.4 \%$; colorectal, $12.8 \%$; and genitourinary, $16.1 \% ; P<.01$ ) (Figure 2). In the IMV group, a similar trend was noted (lung, 29.4\%; breast, 24.7\%; colorectal, 15.1\%; and genitourinary, 19.5\%; $P<.01)$.

\section{Characteristics and Outcomes Across Cancer Subtypes by IPC Use}

In the CCT group, no demographic differences were noted in patients who received IPC services com-

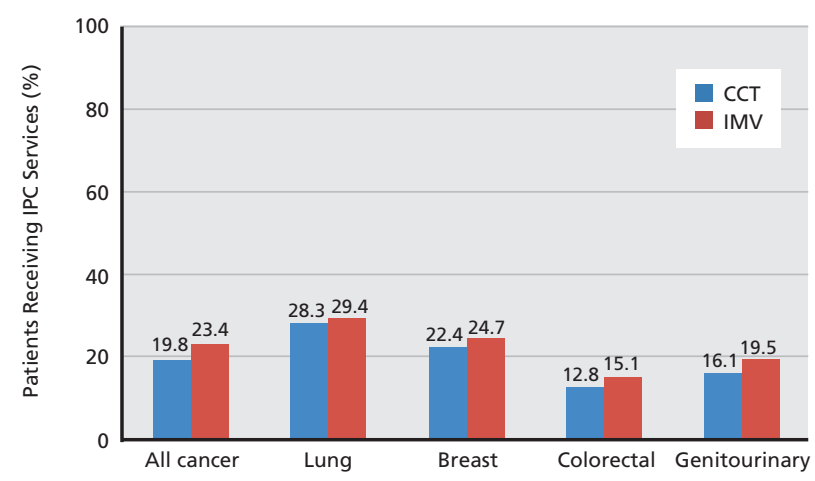

Figure 2. Comparison of inpatient palliative care (IPC) services across cancer subtypes (all $P<.01$ ). The denominator is the number of patients who received critical care therapies (CCT; $n=5,862)$ and invasive mechanical ventilation (IMV; $n=3,035)$. pared with those who did not across all cancer subtypes (Table 3). Among patients with lung, colorectal, or genitourinary cancers, those who used IPC services were more likely to have documented DNR status at time of admission or within 24 hours (lung, $24.8 \%$ vs $16.4 \%$; $P<.01$; colorectal, $24.0 \%$ vs $9.7 \%$; $P<.01$; and genitourinary, $25.0 \%$ vs $10.6 \% ; P<.01$ ) compared with those who did not use IPC services (Table 3). In-hospital mortality was high in patients who received IPC services (range, 51.0\%-75.0\%) and in those who did not receive IPC services across all the examined cancer subtypes (range, 17.6\%49.3\%). Patients with breast cancer who survived and received IPC services had lower cost of care $(\$ 26,530$ vs $\$ 60,861 ; P<.01)$ compared with those who did not receive IPC services. In the IMV group, in-hospital mortality was similarly high in patients who received IPC services (range, 77.6\%-86.3\%) and in those who did not receive IPC services (range, $38.2 \%-59.7 \%$ ) across all examined cancer subtypes (Table 4).

\section{Factors Associated With IPC Use}

In the CCT group, factors associated with IPC use included documented DNR status at time of admission or within 24 hours (adjusted odds ratio [AOR], 2.32; 95\% CI, 1.92-2.71), cancer subtype (colorectal vs lung [ref]: AOR, 0.45; 95\% CI, 0.33-0.56; genitourinary vs lung [ref]: AOR, 0.55; 95\% CI, 0.38-0.71), 
Loh et al

\begin{tabular}{|c|c|c|c|c|}
\hline Characteristics & $\begin{array}{l}\text { All Admissions } \\
(\mathrm{N}=3,035)\end{array}$ & $\begin{array}{c}\text { IPC } \\
(\mathrm{N}=709) \\
\end{array}$ & $\begin{array}{c}\text { No IPC } \\
(\mathrm{N}=2,326)\end{array}$ & $P$ Value \\
\hline Age, n (\%) & & & & .08 \\
\hline$<55 y$ & $537(17.7 \%)$ & $134(18.9 \%)$ & $403(17.3 \%)$ & \\
\hline $55-64 y$ & $716(23.6 \%)$ & $187(26.4 \%)$ & $529(22.7 \%)$ & \\
\hline $65-74 y$ & $864(28.5 \%)$ & $183(25.8 \%)$ & $681(29.3 \%)$ & \\
\hline$\geq 75 y$ & $918(30.3 \%)$ & $205(28.9 \%)$ & $713(30.7 \%)$ & \\
\hline Male sex, $\mathrm{n}(\%)^{\mathrm{a}}$ & $1,544(50.9 \%)$ & $364(51.3 \%)$ & $1,180(50.7 \%)$ & .90 \\
\hline Race, n (\%) & & & & .05 \\
\hline White & $1,689(55.7 \%)$ & $423(59.7 \%)$ & $1,266(54.4 \%)$ & \\
\hline Black & $313(10.3 \%)$ & $59(8.3 \%)$ & $254(10.9 \%)$ & \\
\hline Hispanic & $497(16.4 \%)$ & $105(14.8 \%)$ & $392(16.9 \%)$ & \\
\hline Other $^{b}$ & $395(13.0 \%)$ & $96(13.5 \%)$ & $299(12.9 \%)$ & \\
\hline Missing & $141(4.6 \%)$ & $26(3.7 \%)$ & $115(4.9 \%)$ & \\
\hline Insurance provider, n (\%) & & & & .07 \\
\hline Medicare & $1,731(57.0 \%)$ & $378(53.3 \%)$ & $1,353(58.2 \%)$ & \\
\hline Medicaid & $412(13.6 \%)$ & $97(13.7 \%)$ & $315(13.5 \%)$ & \\
\hline Private & $780(25.7 \%)$ & $204(28.8 \%)$ & $576(24.8 \%)$ & \\
\hline No charge/other & $51(1.7 \%)$ & $17(2.4 \%)$ & $34(1.5 \%)$ & \\
\hline Self-pay & $61(2.0 \%)$ & $13(1.8 \%)$ & $48(2.1 \%)$ & \\
\hline Mean comorbidity score (SD) & $2.3(2)$ & $2.3(1.9)$ & $2.3(2)$ & .47 \\
\hline DNR, $\mathrm{n}(\%)$ & & & & $<.01$ \\
\hline Yes & $471(15.5 \%)$ & $167(23.6 \%)$ & $304(13.1 \%)$ & \\
\hline No & $2,563(84.5 \%)$ & $542(76.4 \%)$ & $2,021(86.9 \%)$ & \\
\hline Missing & $1(0 \%)$ & $0(0 \%)$ & $1(0 \%)$ & \\
\hline Cancer subtype, n (\%) & & & & $<.01$ \\
\hline Lung & $598(19.7 \%)$ & $176(24.8 \%)$ & $422(18.1 \%)$ & \\
\hline Breast & $190(6.3 \%)$ & $47(6.6 \%)$ & $143(6.2 \%)$ & \\
\hline Colorectal & $324(10.7 \%)$ & $49(6.9 \%)$ & $275(11.8 \%)$ & \\
\hline Genitourinary & $262(8.6 \%)$ & $51(7.2 \%)$ & $211(9.1 \%)$ & \\
\hline Other cancer or $>1$ subtype & $905(29.8 \%)$ & $205(28.9 \%)$ & $700(30.1 \%)$ & \\
\hline Not identified & $756(24.9 \%)$ & $181(25.5 \%)$ & $575(24.7 \%)$ & \\
\hline Principal diagnosis, n (\%) & & & & .22 \\
\hline Cancer-related disorders & $1,191(39.2 \%)$ & $264(37.2 \%)$ & $927(39.9 \%)$ & \\
\hline Infections & $887(29.2 \%)$ & $227(32.0 \%)$ & $660(28.4 \%)$ & \\
\hline Other pulmonary disorders & $434(14.3 \%)$ & $111(15.7 \%)$ & $323(13.9 \%)$ & \\
\hline Other cardiovascular and circulation disorders & $166(5.5 \%)$ & $30(4.2 \%)$ & $136(5.9 \%)$ & \\
\hline Other neurologic disorders & $46(1.5 \%)$ & $11(1.6 \%)$ & $35(1.5 \%)$ & \\
\hline Other gastrointestinal disorders & $131(4.3 \%)$ & $27(3.8 \%)$ & $104(4.5 \%)$ & \\
\hline Fluid and electrolyte/renal disorders & $34(1.1 \%)$ & $10(1.4 \%)$ & $24(1.0 \%)$ & \\
\hline Others & $146(4.8 \%)$ & $29(4.1 \%)$ & $117(5.0 \%)$ & \\
\hline Teaching hospital, $\mathrm{n}(\%)^{\mathrm{c}}$ & $1,346(44.3 \%)$ & $325(45.8 \%)$ & $1,021(43.9 \%)$ & .19 \\
\hline Hospital bed count, n (\%) & & & & $<.01$ \\
\hline Small $(<200)$ & $810(26.7 \%)$ & $140(19.8 \%)$ & $670(28.8 \%)$ & \\
\hline Medium (200-399) & $1,283(42.3 \%)$ & $306(43.2 \%)$ & $977(42.0 \%)$ & \\
\hline Large $(\geq 400)$ & $910(30.0 \%)$ & $252(35.5 \%)$ & $658(28.3 \%)$ & \\
\hline Missing & $32(1.0 \%)$ & $11(1.5 \%)$ & $21(0.9 \%)$ & \\
\hline In-hospital mortality, n (\%) & $1,736(57.2 \%)$ & $581(82.0 \%)$ & $1,155(49.7 \%)$ & $<.01$ \\
\hline Discharge among survivors, $\mathrm{n}(\%)$ & & & & .41 \\
\hline Home, home healthcare & $697(53.7 \%)$ & $72(56.3 \%)$ & $625(53.4 \%)$ & \\
\hline SNF, ICF, or facility & $449(34.6 \%)$ & $38(29.7 \%)$ & $411(35.1 \%)$ & \\
\hline Short-term hospital, AMA, or other & $153(11.8 \%)$ & $18(14.1 \%)$ & $135(11.5 \%)$ & \\
\hline Median cost of care among survivors (SD) ${ }^{d}$ & $\$ 67,330(\$ 54,204)$ & $\$ 59,319(\$ 47,859)$ & $\$ 68,132(\$ 54,757)$ & .09 \\
\hline Mean length of stay among survivors (SD), de & $18.2(18.5)$ & $15.6(12.9)$ & $18.5(19.0)$ & .14 \\
\hline
\end{tabular}

Abbreviations: AMA, against medical advice; DNR, do not resuscitate; ICF, intermediate care facility; IMV, invasive mechanical ventilation; IPC, inpatient palliative care services; SNF, skilled nursing facility.

${ }^{\mathrm{a}} 6$ missing.

bincludes Asian or Pacific Islander, Native American, or other as coded on the California State Inpatient Database.

(32 missing.

d 189 missing.

e1 missing.

higher comorbidity score ( 1 vs $\leq 0$ [ref]: $A O R, 1.25$; 95\% CI, 1.00-1.50; $\leq 0$ [ref] vs $\geq 2$ : AOR, 1.41; 95\% CI, 1.15-1.66), infections (vs cancer-related diagnoses [ref]: AOR, 1.25; 95\% CI, 1.03-1.48), and higher bed count (large vs small [ref]: AOR, 1.63; 95\% CI, 1.09-2.16; medium vs small [ref]: AOR, 1.56; 95\% CI, 1.15-1.97) (Table 5). Factors associated with IPC use were similar in the IMV group. 
Inpatient Palliative Care and Cancer

\begin{tabular}{|c|c|c|c|c|c|c|c|c|c|c|c|c|}
\hline \multirow[b]{2}{*}{ Characteristics } & \multicolumn{3}{|c|}{ Lung $(\mathrm{N}=826)$} & \multicolumn{3}{|c|}{ Breast $(\mathrm{N}=\mathbf{2 6 8})$} & \multicolumn{3}{|c|}{ Colorectal $(\mathrm{N}=\mathbf{8 1 3})$} & \multicolumn{3}{|c|}{ Genitourinary $(\mathrm{N}=448)$} \\
\hline & $\underset{\substack{\text { IPC } \\
(n=234)}}{ }$ & $\begin{array}{c}\text { No IPC } \\
(n=592)\end{array}$ & $P$ Value & $\begin{array}{c}\text { IPC } \\
(n=60)\end{array}$ & $\begin{array}{c}\text { No IPC } \\
(\mathrm{n}=208)\end{array}$ & $P$ Value & $\begin{array}{c}\text { IPC } \\
(n=104)\end{array}$ & $\begin{array}{c}\text { No IPC } \\
(n=709)\end{array}$ & $P$ Value & $\begin{array}{c}\text { IPC } \\
(n=72)\end{array}$ & $\begin{array}{c}\text { No IPC } \\
(n=376)\end{array}$ & $P$ Value \\
\hline Age, n (\%) & & & .38 & & & .04 & & & .94 & & & .39 \\
\hline$<55 \mathrm{y}$ & $32(13.7 \%)$ & $77(13.0 \%)$ & & $18(30.0 \%)$ & $58(27.9 \%)$ & & $19(18.3 \%)$ & $126(17.8 \%)$ & & $11(15.3 \%)$ & $35(9.3 \%)$ & \\
\hline $55-64 y$ & $65(27.8 \%)$ & $147(24.8 \%)$ & & $16(26.7 \%)$ & $47(22.6 \%)$ & & $21(20.2 \%)$ & $152(21.4 \%)$ & & $10(13.9 \%)$ & $67(17.8 \%)$ & \\
\hline $65-74 y$ & $88(37.6 \%)$ & $210(35.5 \%)$ & & $7(11.7 \%)$ & $60(28.9 \%)$ & & $25(24.0 \%)$ & $153(21.6 \%)$ & & $16(22.2 \%)$ & $98(26.1 \%)$ & \\
\hline $275 \mathrm{y}$ & $49(20.9 \%)$ & $158(26.7 \%)$ & & $19(31.7 \%)$ & $43(20.7 \%)$ & & $39(37.5 \%)$ & $278(39.2 \%)$ & & $35(48.6 \%)$ & $176(46.8 \%)$ & \\
\hline Male sex, n (\%) & $130(55.6 \%)$ & $318(53.7 \%)$ & .61 & $0(0 \%)$ & $4(1.9 \%)$ & .48 & $58(55.8 \%)$ & $392(55.3 \%)$ & .93 & $58(80.6 \%)$ & $324(86.2 \%)$ & .33 \\
\hline Race, n (\%) & & & .68 & & & .82 & & & .57 & & & .48 \\
\hline White & $148(63.3 \%)$ & $350(59.1 \%)$ & & $36(60.0 \%)$ & $114(54.8 \%)$ & & $57(54.8 \%)$ & $423(59.7 \%)$ & & $47(65.3 \%)$ & $205(54.5 \%)$ & \\
\hline Black & $21(9.0 \%)$ & $56(9.5 \%)$ & & $8(13.3 \%)$ & $23(11.1 \%)$ & & $11(10.6 \%)$ & $66(9.3 \%)$ & & $7(9.7 \%)$ & $49(13 \%)$ & \\
\hline Hispanic & $25(10.7 \%)$ & $64(10.8 \%)$ & & $9(15.0 \%)$ & $42(20.2 \%)$ & & $19(18.3 \%)$ & $109(15.4 \%)$ & & $9(12.5 \%)$ & $74(19.7 \%)$ & \\
\hline Other $^{\text {a }}$ & $32(13.7 \%)$ & $89(15.0 \%)$ & & $6(10.0 \%)$ & $22(10.6 \%)$ & & $14(13.5 \%)$ & $73(10.3 \%)$ & & $6(8.3 \%)$ & $32(8.5 \%)$ & \\
\hline Missing & $8(3.4 \%)$ & $33(5.6 \%)$ & & $1(1.7 \%)$ & $7(3.4 \%)$ & & $3(2.9 \%)$ & $38(5.4 \%)$ & & $3(4.2 \%)$ & $16(4.3 \%)$ & \\
\hline Insurance provider, n (\%) & & & .18 & & & .84 & & & .70 & & & .74 \\
\hline Medicare & $128(54.7 \%)$ & $365(61.7 \%)$ & & $30(50.0 \%)$ & $105(50.5 \%)$ & & $67(64.4 \%)$ & $419(59.1 \%)$ & & $46(63.9 \%)$ & $254(67.6 \%)$ & \\
\hline Medicaid & $29(12.4 \%)$ & $72(12.2 \%)$ & & $9(15.0 \%)$ & $37(17.8 \%)$ & & $9(8.7 \%)$ & $87(12.3 \%)$ & & $9(12.5 \%)$ & $41(10.9 \%)$ & \\
\hline Private & $67(28.6 \%)$ & $134(22.6 \%)$ & & $19(31.7 \%)$ & $62(29.8 \%)$ & & $23(22.1 \%)$ & $178(25.1 \%)$ & & $15(20.8 \%)$ & $75(20.0 \%)$ & \\
\hline No charge/other & $7(3.0 \%)$ & $9(1.5 \%)$ & & $2(3.3 \%)$ & $3(1.4 \%)$ & & $3(2.9 \%)$ & $14(2.0 \%)$ & & $1(1.4 \%)$ & $1(0.3 \%)$ & \\
\hline Self-pay & $3(1.3 \%)$ & $12(2.0 \%)$ & & $0(0 \%)$ & $1(0.5 \%)$ & & $2(1.9 \%)$ & $11(1.6 \%)$ & & $1(1.4 \%)$ & $5(1.3 \%)$ & \\
\hline Mean comorbidity score (SD) & $2.3(1.9)$ & $2.4(1.9)$ & .67 & $2.2(1.8)$ & $2.1(2.0)$ & .73 & $2.2(2.0)$ & $2.0(1.9)$ & .45 & $13.5(12.1)$ & $15.0(16.5)$ & .38 \\
\hline DNR, $\mathrm{n}(\%)$ & & & $<.01^{\mathrm{b}}$ & & & .07 & & & $<.01^{b}$ & & & $<.01^{b}$ \\
\hline Yes & $58(24.8 \%)$ & $97(16.4 \%)$ & & $16(26.7 \%)$ & $34(16.4 \%)$ & & $25(24.0 \%)$ & $69(9.7 \%)$ & & $18(25.0 \%)$ & $40(10.6 \%)$ & \\
\hline No & $176(75.2 \%)$ & $495(83.6 \%)$ & & $44(73.3 \%)$ & $174(83.7 \%)$ & & $79(76.0 \%)$ & $639(90.1 \%)$ & & $54(75.0 \%)$ & $336(89.4 \%)$ & \\
\hline Missing & $0(0 \%)$ & $0(0 \%)$ & & $0(0 \%)$ & $0(0 \%)$ & & $0(0 \%)$ & $1(0.1 \%)$ & & $0(0 \%)$ & $0(0 \%)$ & \\
\hline Principal diagnosis, $\mathrm{n}(\%)$ & & & .20 & & & .49 & & & $<.01^{\mathrm{b}}$ & & & .75 \\
\hline Cancer-related disorders & $96(41.0 \%)$ & $213(36.0 \%)$ & & $17(28.3 \%)$ & $50(24.0 \%)$ & & $54(51.9 \%)$ & $460(64.9 \%)$ & & $19(26.4 \%)$ & $136(36.2 \%)$ & \\
\hline Infections & $60(25.6 \%)$ & $150(25.3 \%)$ & & $22(36.7 \%)$ & $61(29.3 \%)$ & & $27(26.0 \%)$ & $86(12.1 \%)$ & & $24(33.3 \%)$ & $103(27.4 \%)$ & \\
\hline Other pulmonary disorders & $44(18.8 \%)$ & $98(16.6 \%)$ & & $5(8.3 \%)$ & $27(13.0 \%)$ & & $5(4.8 \%)$ & $15(2.1 \%)$ & & $6(8.3 \%)$ & $38(10.1 \%)$ & \\
\hline $\begin{array}{l}\text { Other cardiovascular and } \\
\text { circulation disorders }\end{array}$ & $8(3.4 \%)$ & $34(5.7 \%)$ & & $2(3.3 \%)$ & $11(5.3 \%)$ & & $3(2.9 \%)$ & $20(2.8 \%)$ & & $5(6.9 \%)$ & $23(6.1 \%)$ & \\
\hline Other neurologic disorders & $4(1.7 \%)$ & $9(1.5 \%)$ & & $2(3.3 \%)$ & $8(3.9 \%)$ & & $2(1.9 \%)$ & $7(1.0 \%)$ & & $0(0 \%)$ & $3(0.8 \%)$ & \\
\hline $\begin{array}{l}\text { Other gastrointestinal } \\
\text { disorders }\end{array}$ & $13(5.6 \%)$ & $38(6.4 \%)$ & & $2(3.3 \%)$ & $21(10.1 \%)$ & & $8(7.7 \%)$ & $67(9.5 \%)$ & & $8(11.1 \%)$ & $30(8.0 \%)$ & \\
\hline $\begin{array}{l}\text { Fluid and electrolyte/renal } \\
\text { disorders }\end{array}$ & $5(2.1 \%)$ & $16(2.7 \%)$ & & $5(8.3 \%)$ & $9(4.3 \%)$ & & $2(1.9 \%)$ & $15(2.1 \%)$ & & $6(8.3 \%)$ & $27(7.2 \%)$ & \\
\hline Others & $4(1.7 \%)$ & $34(5.7 \%)$ & & $5(8.3 \%)$ & $21(10.1 \%)$ & & $3(2.9 \%)$ & $39(5.5 \%)$ & & $4(5.6 \%)$ & $16(4.3 \%)$ & \\
\hline Teaching hospital, n (\%) & $88(37.6 \%)$ & $240(40.5 \%)$ & .50 & $27(45.0 \%)$ & $89(42.8 \%)$ & .86 & $40(38.5 \%)$ & $288(40.6 \%)$ & .66 & $32(44.4 \%)$ & $172(45.7 \%)$ & .98 \\
\hline Hospital bed count, n (\%) & & & $<.01^{\mathrm{b}}$ & & & & .43 & & .61 & & & .09 \\
\hline Small $(<200)$ & $47(20.1 \%)$ & $186(31.4 \%)$ & & $11(18.3 \%)$ & $60(28.9 \%)$ & & $33(31.7 \%)$ & $213(30.0 \%)$ & & $15(20.8 \%)$ & $113(30.1 \%)$ & \\
\hline Medium (200-399) & $110(47.0 \%)$ & $255(43.1 \%)$ & & $31(51.7 \%)$ & $91(43.8 \%)$ & & $39(37.5 \%)$ & $306(43.2 \%)$ & & $26(36.1 \%)$ & $156(41.5 \%)$ & \\
\hline Large $(\geq 400)$ & $76(32.5 \%)$ & $145(24.5 \%)$ & & $16(26.7 \%)$ & $52(25.0 \%)$ & & $30(28.9 \%)$ & $183(25.8 \%)$ & & $30(41.7 \%)$ & $102(27.1 \%)$ & \\
\hline In-hospital mortality, n (\%) & $169(72.2 \%)$ & $292(49.3 \%)$ & $<.01^{b}$ & $45(75.0 \%)$ & $94(45.2 \%)$ & $<.01^{\mathrm{b}}$ & $53(51.0 \%)$ & $125(17.6 \%)$ & $<.01^{b}$ & $46(63.9 \%)$ & $135(35.9 \%)$ & $<.01^{\mathrm{b}}$ \\
\hline \multicolumn{2}{|l|}{ Discharge among survivors, $\mathrm{n}(\%)$} & & .25 & & & .84 & & & .50 & & & .10 \\
\hline Home, home healthcare & $43(66.2 \%)$ & $170(56.7 \%)$ & & $10(66.7 \%)$ & $68(59.7 \%)$ & & $36(70.6 \%)$ & $388(66.4 \%)$ & & $15(57.7 \%)$ & $140(58.1 \%)$ & \\
\hline SNF, ICF, or facility & $8(12.3 \%)$ & $34(11.3 \%)$ & & $1(6.7 \%)$ & $7(6.1 \%)$ & & $5(9.8 \%)$ & $42(7.2 \%)$ & & $5(19.2 \%)$ & $18(7.5 \%)$ & \\
\hline $\begin{array}{l}\text { Short-term hospital, AMA, } \\
\text { or other }\end{array}$ & $14(21.5 \%)$ & $96(32.0 \%)$ & & $4(26.7 \%)$ & $39(34.2 \%)$ & & $10(19.6 \%)$ & $154(26.4 \%)$ & & $6(23.1 \%)$ & $83(34.4 \%)$ & \\
\hline $\begin{array}{l}\text { Median cost of care among } \\
\text { survivors (SD) }\end{array}$ & $\begin{array}{l}\$ 42,077 \\
(\$ 35,359)\end{array}$ & $\begin{array}{c}\$ 48,903 \\
(\$ 46,236)\end{array}$ & .42 & $\begin{array}{l}\$ 26,530 \\
(\$ 23,415)\end{array}$ & $\begin{array}{c}\$ 60,861 \\
(\$ 60,463)\end{array}$ & $<.01^{b}$ & $\begin{array}{l}\$ 48,196 \\
(\$ 34,900)\end{array}$ & $\begin{array}{l}\$ 54,840 \\
(\$ 43,776)\end{array}$ & .37 & $\begin{array}{c}\$ 54,569 \\
(\$ 44,188)\end{array}$ & $\begin{array}{l}\$ 52,291 \\
(\$ 40,549)\end{array}$ & .99 \\
\hline $\begin{array}{l}\text { Mean length of stay among } \\
\text { survivors (SD), d }\end{array}$ & $13.6(11.9)$ & $15.2(17.0)$ & .83 & $8.9(6.9)$ & $17.9(16.0)$ & .02 & $16.0(11.8)$ & $17.0(11.9)$ & .59 & $16.4(12.7)$ & $15.5(10.6)$ & .75 \\
\hline
\end{tabular}

Abbreviations: AMA, against medical advice; CCT, critical care therapies; DNR, do not resuscitate; ICF, intermediate care facility; IPC, inpatient palliative care services; SNF, skilled nursing facility. ancludes Asian or Pacific Islander, Native American, or other as coded on the California State Inpatient Database.

bTests were considered significant if $P<.0125$ after adjusting for multiple comparisons.

\section{Discussion}

In this retrospective observational cohort of $>5,500$ critically ill hospitalized patients with metastatic cancer, we found that only $20 \%$ used IPC services. In-hospital mortality was much higher in those who received IPC services. In survivors, the LOS and cost of care were lower in those who received IPC services compared with those who did not. Although use of IPC services varied across cancer subtypes, it was more frequent in lung cancer (28.3\%), followed by breast $(22.4 \%)$, colorectal $(12.8 \%)$, and genitourinary $(16.1 \%)$, but it remained low overall. The differential use of IPC may be due to prior studies demonstrating benefits of palliative care services for patients with lung cancer. ${ }^{7}$ We also identified several factors associated with IPC use, including documented DNR status at time of admission or within 24 hours, lung cancer, higher comorbidity, hospital admission with infections, and admission to hospitals with a higher bed count. 
Loh et al

\begin{tabular}{|c|c|c|c|c|c|c|c|c|c|c|c|c|}
\hline \multirow[b]{2}{*}{ Characteristics } & \multicolumn{3}{|c|}{ Lung ( $N=598)$} & \multicolumn{3}{|c|}{ Breast $(\mathrm{N}=190)$} & \multicolumn{3}{|c|}{ Colorectal $(\mathrm{N}=324)$} & \multicolumn{3}{|c|}{ Genitourinary $(\mathrm{N}=262)$} \\
\hline & $\begin{array}{c}\text { IPC } \\
(n=176)\end{array}$ & $\begin{array}{c}\text { No IPC } \\
(\mathrm{n}=422)\end{array}$ & $P$ Value & $\begin{array}{c}\text { IPC } \\
(n=47)\end{array}$ & $\begin{array}{c}\text { No IPC } \\
(n=143)\end{array}$ & $P$ Value & $\begin{array}{c}\text { IPC } \\
(n=49)\end{array}$ & $\begin{array}{c}\text { No IPC } \\
(n=275)\end{array}$ & $P$ Value & $\begin{array}{c}\text { IPC } \\
(n=51)\end{array}$ & $\begin{array}{c}\text { No IPC } \\
(\mathrm{n}=\mathbf{2 1 1})\end{array}$ & $P$ Value \\
\hline Age, $\mathrm{n}(\%)$ & & & .64 & & & .10 & & & .36 & & & .33 \\
\hline$<55 y$ & $23(13.1 \%)$ & $59(14.0 \%)$ & & $16(34.0 \%)$ & $44(30.8 \%)$ & & $8(16.3 \%)$ & $43(15.6 \%)$ & & $9(17.7 \%)$ & $19(9.0 \%)$ & \\
\hline $55-64 y$ & $49(27.8 \%)$ & $106(25.1 \%)$ & & $14(29.8 \%)$ & $34(23.8 \%)$ & & $14(28.6 \%)$ & $50(18.2 \%)$ & & $9(17.7 \%)$ & $35(16.6 \%)$ & \\
\hline $65-74 y$ & $66(37.5 \%)$ & $147(34.8 \%)$ & & $5(10.6 \%)$ & $40(28.0 \%)$ & & $9(18.4 \%)$ & $67(24.4 \%)$ & & $11(21.6 \%)$ & $53(25.1 \%)$ & \\
\hline$\geq 75 y$ & $38(21.6 \%)$ & $110(26.1 \%)$ & & $12(25.5 \%)$ & $25(17.5 \%)$ & & $18(36.7 \%)$ & $115(41.8 \%)$ & & $22(43.1 \%)$ & $104(49.3 \%)$ & \\
\hline Male sex, n (\%) & $98(55.7 \%)$ & $228(54.0 \%)$ & .63 & $0(0 \%)$ & $3(2.1 \%)$ & .51 & $30(61.2 \%)$ & $158(57.5 \%)$ & .62 & $41(80.4 \%)$ & $186(88.2 \%)$ & .26 \\
\hline Race, n (\%) & & & .64 & & & .65 & & & .66 & & & .43 \\
\hline White & $114(64.8 \%)$ & $246(58.3 \%)$ & & $27(57.5 \%)$ & $75(52.5 \%)$ & & $24(49.0 \%)$ & $152(55.3 \%)$ & & $33(64.7 \%)$ & $105(49.8 \%)$ & \\
\hline Black & $14(8.0 \%)$ & $45(10.7 \%)$ & & $8(17.0 \%)$ & $17(11.9 \%)$ & & $3(6.1 \%)$ & $28(10 . \%)$ & & $5(9.8 \%)$ & $30(14.2 \%)$ & \\
\hline Hispanic & $17(9.7 \%)$ & $47(11.1 \%)$ & & $6(12.8 \%)$ & $28(19.6 \%)$ & & $11(22.5 \%)$ & $46(16.7 \%)$ & & $8(15.7 \%)$ & $42(19.9 \%)$ & \\
\hline Other $^{a}$ & $24(13.6 \%)$ & $62(14.7 \%)$ & & $5(10.6 \%)$ & $16(11.2 \%)$ & & $8(16.3 \%)$ & $33(12.0 \%)$ & & $3(5.9 \%)$ & $22(10.4 \%)$ & \\
\hline Missing & $7(4.0 \%)$ & $22(5.2 \%)$ & & $1(2.1 \%)$ & $7(4.9 \%)$ & & $3(6.1 \%)$ & $16(5.8 \%)$ & & $2(3.9 \%)$ & $12(5.7 \%)$ & \\
\hline Insurance provider, n (\%) & & & .32 & & & .57 & & & .50 & & & .64 \\
\hline Medicare & $97(55.1 \%)$ & $262(62.1 \%)$ & & $19(40.4 \%)$ & $68(47.6 \%)$ & & $27(55.1 \%)$ & $170(61.8 \%)$ & & $31(60.8 \%)$ & $144(68.3 \%)$ & \\
\hline Medicaid & $22(12.5 \%)$ & $52(12.3 \%)$ & & $8(17.0 \%)$ & $31(21.7 \%)$ & & $6(12.2 \%)$ & $32(11.6 \%)$ & & $8(15.7 \%)$ & $22(10.4 \%)$ & \\
\hline Private & $50(28.4 \%)$ & $91(21.6 \%)$ & & $18(38.3 \%)$ & $40(28.0 \%)$ & & $12(24.5 \%)$ & $64(23.3 \%)$ & & $10(19.6 \%)$ & $40(19.0 \%)$ & \\
\hline No charge/other & $5(2.8 \%)$ & $8(1.9 \%)$ & & $2(4.3 \%)$ & $3(2.1 \%)$ & & $2(4.1 \%)$ & $3(1.1 \%)$ & & $1(2.0 \%)$ & $1(0.5 \%)$ & \\
\hline Self-pay & $2(1.1 \%)$ & $9(2.1 \%)$ & & $0(0 \%)$ & $1(0.7 \%)$ & & $2(4.1 \%)$ & $6(2.2 \%)$ & & $1(2.0 \%)$ & $4(1.9 \%)$ & \\
\hline Mean comorbidity score (SD) & $2.3(1.8)$ & $2.4(1.9)$ & .79 & $2.3(1.9)$ & $2.2(2.1)$ & .65 & $2.4(2.2)$ & $2.5(2)$ & .49 & $2.9(2)$ & $2.9(2.1)$ & .84 \\
\hline DNR, n (\%) & & & $.01^{\mathrm{b}}$ & & & .15 & & & .03 & & & .05 \\
\hline Yes & $41(23.3 \%)$ & $63(14.9 \%)$ & & $12(25.5 \%)$ & $23(16.1 \%)$ & & $12(24.5 \%)$ & $30(10.9 \%)$ & & $11(21.6 \%)$ & $24(11.4 \%)$ & \\
\hline No & $135(76.7 \%)$ & $359(85.1 \%)$ & & $35(74.5 \%)$ & $120(83.9 \%)$ & & $37(75.5 \%)$ & $244(88.7 \%)$ & & $40(78.4 \%)$ & $187(88.6 \%)$ & \\
\hline Missing & $0(0 \%)$ & $0(0 \%)$ & & $0(0 \%)$ & $0(0 \%)$ & & $0(0 \%)$ & $1(0.4 \%)$ & & $0(0 \%)$ & $0(0 \%)$ & \\
\hline Principal diagnosis, n (\%) & & & .21 & & & .10 & & & .23 & & & .56 \\
\hline Cancer-related disorders & $71(40.3 \%)$ & $151(35.8 \%)$ & & $14(29.8 \%)$ & $31(21.7 \%)$ & & $19(38.8 \%)$ & $148(53.8 \%)$ & & $11(21.6 \%)$ & $55(26.1 \%)$ & \\
\hline Infections & $50(28.4 \%)$ & $121(28.7 \%)$ & & $20(42.6 \%)$ & $53(37.1 \%)$ & & $17(34.7 \%)$ & $65(23.6 \%)$ & & $22(43.1 \%)$ & $78(37.0 \%)$ & \\
\hline Other pulmonary disorders & $41(23.3 \%)$ & $87(20.6 \%)$ & & $5(10.6 \%)$ & $24(16.8 \%)$ & & $5(10.2 \%)$ & $15(5.5 \%)$ & & $5(9.8 \%)$ & $35(16.6 \%)$ & \\
\hline $\begin{array}{l}\text { Other cardiovascular and } \\
\text { circulation disorders }\end{array}$ & $2(1.1 \%)$ & $27(6.4 \%)$ & & $1(2.1 \%)$ & $10(7.0 \%)$ & & $3(6.1 \%)$ & $12(4.4 \%)$ & & $3(5.9 \%)$ & $17(8.1 \%)$ & \\
\hline Other neurologic disorders & $4(2.3 \%)$ & $7(1.7 \%)$ & & $1(2.1 \%)$ & $5(3.5 \%)$ & & $2(4.1 \%)$ & $3(1.1 \%)$ & & $0(0 \%)$ & $1(0.5 \%)$ & \\
\hline $\begin{array}{l}\text { Other gastrointestinal } \\
\text { disorders }\end{array}$ & $4(2.3 \%)$ & $12(2.8 \%)$ & & $0(0 \%)$ & $8(5.6 \%)$ & & $2(4.1 \%)$ & $18(6.6 \%)$ & & $5(9.8 \%)$ & $13(6.2 \%)$ & \\
\hline $\begin{array}{l}\text { Fluid and electrolyte/renal } \\
\text { disorders }\end{array}$ & $1(0.6 \%)$ & $4(1.0 \%)$ & & $3(6.4 \%)$ & $1(0.7 \%)$ & & $0(0 \%)$ & $1(0.4 \%)$ & & $2(3.9 \%)$ & $2(0.9 \%)$ & \\
\hline Others & $3(1.7 \%)$ & $13(3.1 \%)$ & & $3(6.4 \%)$ & $11(7.7 \%)$ & & $1(2.0 \%)$ & $13(4.7 \%)$ & & $3(5.9 \%)$ & $10(4.7 \%)$ & \\
\hline Teaching hospital, n (\%) & $73(41.5 \%)$ & $175(41.5 \%)$ & .90 & $24(51.1 \%)$ & $57(39.9 \%)$ & .39 & $22(44.9 \%)$ & $106(38.5 \%)$ & .56 & $23(45.1 \%)$ & $91(43.1 \%)$ & .86 \\
\hline Hospital bed count, $n(\%)$ & & & $.01^{\mathrm{b}}$ & & & .74 & & & 11 & & & $<.01^{\mathrm{b}}$ \\
\hline Small $(<200)$ & $37(21.0 \%)$ & $137(32.5 \%)$ & & $10(21.3 \%)$ & $40(28.0 \%)$ & & $13(26.5 \%)$ & $90(32.7 \%)$ & & $10(19.6 \%)$ & $65(30.8 \%)$ & \\
\hline Medium (200-399) & $78(44.3 \%)$ & $181(42.9 \%)$ & & $24(51.1 \%)$ & $68(47.6 \%)$ & & $16(32.7 \%)$ & $115(41.8 \%)$ & & $17(33.3 \%)$ & $96(45.5 \%)$ & \\
\hline Large $(\geq 400)$ & $60(34.1 \%)$ & $100(23.7 \%)$ & & $12(25.5 \%)$ & $30(21.0 \%)$ & & $20(40.8 \%)$ & $67(24.4 \%)$ & & $24(47.1 \%)$ & $49(23.2 \%)$ & \\
\hline Missing & $1(0.6 \%)$ & $4(0.9 \%)$ & & $2(2.1 \%)$ & $5(3.4 \%)$ & & $0(0 \%)$ & $3(1.0 \%)$ & & $0(0 \%)$ & $1(0.5 \%)$ & \\
\hline In-hospital mortality, n (\%) & $144(81.8 \%)$ & $252(59.7 \%)$ & $<.01^{\mathrm{b}}$ & $39(83.0 \%)$ & $85(59.4 \%)$ & $<.01^{\mathrm{b}}$ & $38(77.6 \%)$ & $105(38.2 \%)$ & $<.01^{\mathrm{b}}$ & $44(86.3 \%)$ & $112(53.1 \%)$ & $<.01^{\mathrm{b}}$ \\
\hline Discharge among survivors, $\mathrm{n}$ & & & .45 & & & .98 & & & .63 & & & .06 \\
\hline Home, home healthcare & $20(62.5 \%)$ & $89(52.4 \%)$ & & $4(50.0 \%)$ & $29(50.0 \%)$ & & $6(54.6 \%)$ & $86(50.6 \%)$ & & $3(42.9 \%)$ & $45(45.5 \%)$ & \\
\hline SNF, ICF, or facility & $5(15.6 \%)$ & $25(14.7 \%)$ & & $1(12.5 \%)$ & $6(10.3 \%)$ & & $2(18.2 \%)$ & $18(10.6 \%)$ & & $3(42.9 \%)$ & $12(12.1 \%)$ & \\
\hline $\begin{array}{l}\text { Short-term hospital, AMA, } \\
\text { or other }\end{array}$ & $7(21.9 \%)$ & $56(32.9 \%)$ & & $3(37.5 \%)$ & $23(39.7 \%)$ & & $3(27.3 \%)$ & $66(38.8 \%)$ & & $1(14.3 \%)$ & $42(42.4 \%)$ & \\
\hline $\begin{array}{l}\text { Mean cost of care among } \\
\text { survivors (SD) }\end{array}$ & $\begin{array}{l}\$ 51,040 \\
(\$ 40,801)\end{array}$ & $\begin{array}{c}\$ 58,839 \\
(\$ 46,218)\end{array}$ & .33 & $\begin{array}{c}\$ 35,709 \\
(\$ 29,466)\end{array}$ & $\begin{array}{l}\$ 75,353 \\
(\$ 71,486)\end{array}$ & .05 & $\begin{array}{l}\$ 74,061 \\
(\$ 45,663)\end{array}$ & $\begin{array}{c}\$ 76,487 \\
(\$ 56,100)\end{array}$ & .80 & $\begin{array}{c}\$ 74,303 \\
(\$ 62,549)\end{array}$ & $\begin{array}{l}\$ 63,495 \\
(\$ 46,015)\end{array}$ & .97 \\
\hline $\begin{array}{l}\text { Mean length of stay among } \\
\text { survivors (SD), } d\end{array}$ & $16.0(15.5)$ & $16.7(18.2)$ & .75 & $10.0(7.1)$ & $20.1(18.6)$ & .14 & $16.6(11.6)$ & $20.1(14.7)$ & .47 & $20.6(19.1)$ & $15.6(10.5)$ & .70 \\
\hline
\end{tabular}

Abbreviations: AMA, against medical advice; DNR, do not resuscitate; ICF, intermediate care facility; IMV, invasive mechanical ventilation; IPC, inpatient palliative care services; SNF, skilled nursing facility. Includes Asian or Pacific Islander, Native American, or other as coded on the California State Inpatient Database.

${ }^{\text {In }}$ Tests were considered significant if $P<.0125$ after adjusting for multiple comparisons.
.

Given the retrospective design of the study, the higher in-hospital mortality rate in patients who received IPC services does not indicate a causal relationship between IPC use and mortality given the difficulty in adjusting for all potential confounders. Rather, it suggests that IPC services were provided to those whom clinicians perceived were likely to die and those who were sicker. Despite this, at least a quarter of patients who received CCTs died without receiving any IPC services. These findings suggest a clear opportunity to improve care for this vulnerable population. Earlier incorporation of IPC services may help in assessing patient preferences for life-prolonging treatment at the end of life and in redirecting care to fit patient preferences.

In our cohort, LOS and cost of care were also lower in the group that had received IPC services. When we evaluated outcomes based on IPC use in the various cancer subtypes, only patients with breast cancer who survived had statistically lower 


\begin{tabular}{|c|c|c|}
\hline \multirow[b]{2}{*}{ Variables } & \multicolumn{2}{|c|}{ Adjusted Odd Ratios (95\% Cl) } \\
\hline & CCTs & IMV \\
\hline \multicolumn{3}{|l|}{ Age } \\
\hline$<55 y$ & 1 (Ref) & 1 (Ref) \\
\hline $55-64$ y & $1.00(0.79-1.21)$ & $1.05(0.77-1.32)$ \\
\hline $65-74 y$ & $0.93(0.70-1.16)$ & $1.00(0.69-1.31)$ \\
\hline$\geq 75 \mathrm{y}$ & $0.92(0.69-1.16)$ & $1.08(0.73-1.42)$ \\
\hline \multicolumn{3}{|l|}{ Sex } \\
\hline Male & 1 (Ref) & 1 (Ref) \\
\hline Female & $0.97(0.84-1.11)$ & $0.93(0.76-1.10)$ \\
\hline \multicolumn{3}{|l|}{ Race } \\
\hline White & 1 (Ref) & 1 (Ref) \\
\hline Hispanic & $1.02(0.82-1.22)$ & $0.94(0.71-1.17)$ \\
\hline Black & $1.03(0.77-1.29)$ & $0.81(0.56-1.07)$ \\
\hline Othera & $1.07(0.85-0.29)$ & $0.97(0.71-1.22)$ \\
\hline \multicolumn{3}{|l|}{ Insurance provider } \\
\hline Medicare & 1 (Ref) & 1 (Ref) \\
\hline Medicaid & $0.91(0.68-1.15)$ & $1.17(0.80-0.95)$ \\
\hline Private & $1.02(0.81-1.24)$ & $1.29(9.05-1.63)$ \\
\hline No charge/other & $1.80(0.86-2.74)$ & $2.32(0.84-3.80)$ \\
\hline Self-pay & $0.81(0.35-1.27)$ & $1.13(0.41-1.85)$ \\
\hline \multicolumn{3}{|l|}{ Comorbidity score } \\
\hline$\leq 0$ & 1 (Ref) & 1 (Ref) \\
\hline 1 & $1.25(1.00-1.50)$ & $1.18(0.85-1.50)$ \\
\hline$\geq 2$ & $1.41(1.15-1.66)$ & $1.40(1.05-1.74)$ \\
\hline \multicolumn{3}{|l|}{ DNR } \\
\hline No & 1 (Ref) & 1 (Ref) \\
\hline Yes & $2.32(1.92-2.71)$ & $1.97(1.54-2.41)$ \\
\hline \multicolumn{3}{|l|}{ Cancer subtype } \\
\hline Lung & 1 (Ref) & 1 (Ref) \\
\hline Not identified & $0.70(0.55-0.84)$ & $0.71(0.53-0.89)$ \\
\hline Breast & $0.77(0.51-1.04)$ & $0.96(0.59-1.33)$ \\
\hline Colorectal & $0.45(0.33-0.56)$ & $0.51(0.33-0.68)$ \\
\hline Genitourinary & $0.55(0.38-0.71)$ & $0.67(0.43-0.91)$ \\
\hline $\begin{array}{l}\text { Other cancer or }>2 \\
\text { subtypes }\end{array}$ & $0.66(0.53-0.79)$ & $0.76(0.57-0.94)$ \\
\hline \multicolumn{3}{|l|}{ Principal diagnosis } \\
\hline Cancer-related disorders & 1 (Ref) & 1 (Ref) \\
\hline Infections & $1.25(1.03-1.48)$ & $1.19(0.93-1.45)$ \\
\hline Other pulmonary disorders & $1.14(0.87-1.42)$ & $1.12(0.81-1.42)$ \\
\hline $\begin{array}{l}\text { Other cardiovascular and } \\
\text { circulation disorders }\end{array}$ & $0.98(0.64-1.32)$ & $0.80(0.46-1.13)$ \\
\hline $\begin{array}{l}\text { Other neurologic and } \\
\text { psychiatric disorders }\end{array}$ & $0.95(0.37-1.53)$ & $0.88(0.25-1.51)$ \\
\hline $\begin{array}{l}\text { Other gastrointestinal } \\
\text { disorders }\end{array}$ & $0.91(0.68-1.14)$ & $0.90(0.49-1.32)$ \\
\hline $\begin{array}{l}\text { Fluid and electrolyte/renal } \\
\text { disorders }\end{array}$ & $1.02(0.65-1.39)$ & $1.70(0.44-2.97)$ \\
\hline Others & $0.76(0.52-1.00)$ & $0.90(0.49-1.32)$ \\
\hline Not a teaching hospital & $1.02(0.77-1.27)$ & $1.08(0.74-1.42)$ \\
\hline \multicolumn{3}{|l|}{ Hospital bed count } \\
\hline Small $(<200)$ & 1 (Ref) & 1 (Ref) \\
\hline Medium (200-399) & $1.56(1.15-1.97)$ & $1.69(1.11-2.27)$ \\
\hline Large $(\geq 400)$ & $1.63(1.09-2.16)$ & $1.83(1.04-2.62)$ \\
\hline
\end{tabular}

Abbreviations: CCTs, critical care therapies; DNR, do not resuscitate; IMV, invasive mechanical ventilation; IPC, inpatient palliative care.

alncludes Asian or Pacific Islander, Native American, or other as coded on the California State Inpatient Database.

cost of care compared with other cancer subtypes. It is worth noting that patients with lung and genitourinary cancers who received IPC services also had lower cost of care, but these were not statistically significant likely due to the same population size. In terms of place of death, Paris and Morrison ${ }^{18}$ demonstrated that IPC services led to a significant reduction in the odds of dying in a hospital for patients with advanced gastrointestinal cancers. A similar proportion of patients were discharged to home in our study, but we are unable to determine which of these patients were discharged home on hospice, because the CA SID does not include these data. Early IPC interventions implemented prospectively in the ICU have also been shown to result in a more frequent change of code status to DNR and increased rate of hospice referral, as well as significant reduction in LOS and cost of care. ${ }^{19}$

Prior studies have shown a discrepancy between patient preference for end-of-life care and the reality of practice. ${ }^{20,21}$ Most patients who are terminally ill from cancer report a preference to die at home, yet many of them receive CCTs, and many end up dying in the hospital. ${ }^{22}$ It is possible that this disparity could be reduced by increased use of outpatient and/or inpatient palliative care and hospice services, although this needs to be further investigated. ${ }^{18}$ Professional organizations such as ASCO recommend that patients with metastatic disease have palliative care services integrated early in their treatment course and throughout their illness ${ }^{23}$; the impact on the quality of end-of-life care has been shown to be greater when palliative services are initiated earlier, specifically in the outpatient setting., ${ }^{9,24}$ However, data on how the timing of palliative care affects quality and costs remain limited. Still, IPC services can complement the care of these patients when admitted to the hospital, because preferences for endof-life care often change. For example, in patients on chronic dialysis (not specific to cancer), 45\% had their care changed from aggressive to palliative when they were admitted to a hospital. ${ }^{25}$

Our study has several limitations. First, we only included specific CCTs and our findings therefore do not apply to other types of therapies, such as jejunostomy or vasopressors. Second, information on socioeconomic status (eg, marital status, education, ZIP code) is not available in the CA SID. Third, we were unable to measure the use of outpatient palliative care services because this variable was not captured in the CA SID. Hospice information was also not available in the CA SID, and therefore we were unable to comment on patients who were discharged to hospice. Fourth, this study is limited by a lack of 
information on mortality after discharge. We also do not have data on the date of IPC service use. Fifth, IPC service use was determined based on ICD-9 codes, specifically a $\mathrm{V}$ code, and may underestimate the actual use of IPC services. Therefore, we may not have captured some patients who received IPC services, but when the ICD-9 code V66.7 was identified, the patient most likely received IPC services. It should be noted that although the presence of ICD9 code V66.7 indicated involvement of IPC services, the nature of this involvement was unclear (eg, palliative care consult, admission to the palliative care units or reasons for involvement). Although this code may not have been used each time IPC services were involved, the use pattern of this code would theoretically be consistent across cancer subtypes. Sixth, we could not determine the number of hospitals with IPC services, the IPC team structure, and their capacity to see patients. Seventh, the CA SID defined DNR status as present if this was docu- mented at time of admission or within 24 hours. It is possible that IPC service was involved in the first 24 hours of admission, which may explain why more patients who received IPC services had DNR status documented more frequently. Lastly, it is unclear if the information on charges available from the CA SID was comprehensive of all services provided in the hospital.

\section{Conclusions}

Use of IPC services was low in this cohort of patients with metastatic cancer who received CCTs despite poor prognosis and high in-hospital mortality. More studies are needed to understand motivators behind the use of IPC services (eg, whether clinicians tend to use IPC in patients with predicted poorer short-term outcomes) and to assess the impact early IPC use can have on patient care.

\section{References}

1. American Cancer Society. Cancer Facts \& Figures 2017. Atlanta, GA: American Cancer Society; 2017.

2. Miller KD, Siegel RL, Lin CC, et al. Cancer treatment and survivorship statistics, 2016. CA Cancer J Clin 2016;66:271-289.

3. Azoulay E, Schellongowski P, Darmon M, et al. The intensive care medicine research agenda on critically ill oncology and hematology patients. Intensive Care Med 2017;43:1366-1382.

4. Loh KP, Kansagra A, Shieh MS, et al. Predictors of in-hospital mortality in patients with metastatic cancer receiving specific critical care therapies. J Natl Compr Cancer Netw 2016;14:979-987.

5. Loh KP, Kansagra A, Shieh MS, et al. Predictors of the use of specific critical care therapies in patients with metastatic cancer. J Natl Compr Cancer Netw 2017;15:22-30.

6. Khandelwal N, Kross EK, Engelberg RA, et al. Estimating the effect of palliative care interventions and advance care planning on ICU utilization: a systematic review. Crit Care Med 2015;43:1102-1111.

7. Temel JS, Greer JA, Muzikansky A, et al. Early palliative care for patients with metastatic non-small-cell lung cancer. N Engl J Med 2010;363:733742.

8. May P, Garrido MM, Cassel JB, et al. Palliative care teams' cost-saving effect is larger for cancer patients with higher numbers of comorbidities. Health Aff (Millwood) 2016;35:44-53.

9. Scibetta C, Kerr K, Mcguire J, Rabow MW. The costs of waiting: implications of the timing of palliative care consultation among a cohort of decedents at a comprehensive cancer center. J Palliat Med 2016;19:69-75.

10. Mulvey CL, Smith TJ, Gourin CG. The use of inpatient palliative care services in patients with metastatic incurable head and neck cancer. Head Neck 2016;38:355-363.

11. O'Connor NR, Moyer ME, Behta M, Casarett DJ. The impact of inpatient palliative care consultations on 30-day hospital readmissions. J Palliat Med 2015;18:956-961.

12. Weiner MG, Livshits A, Carozzoni $C$, et al. Derivation of malignancy status from ICD-9 codes. AMIA Annu Symp Proc 2003;2003:1050.

13. Eichler AF, Lamont EB. Utility of administrative claims data for the study of brain metastases: a validation study. J Neurooncol 2009;95:427-431.
14. Gagne JJ, Glynn RJ, Avorn J, et al. A combined comorbidity score predicted mortality in elderly patients better than existing scores. J Clin Epidemiol 2011;64:749-759.

15. Hua M, Li G, Clancy C, et al. Validation of the V66.7 code for palliative care consultation in a single academic medical center. J Palliat Med 2016;20:372-377.

16. Feder SL, Tate J, Womack J, et al. Validation of the diagnostic code for palliative care in patients hospitalized with heart failure within the Department of Veterans Affairs (S752). J Pain Symptom Manage 2017;53:438-439

17. Cost-to-Charge Ratio Files. Healthcare Cost and Utilization Project (HCUP). June 2018. Agency for Healthcare Research and Quality, Rockville, MD. Available at: www.hcup-us.ahrq.gov/db/state/costtocharge. jsp. Accessed September 19, 2015.

18. Paris J, Morrison RS. Evaluating the effects of inpatient palliative care consultations on subsequent hospice use and place of death in patients with advanced GI cancers. J Oncol Pract 2014;10:174-177.

19. Zalenski RJ, Jones SS, Courage $C$, et al. Impact of palliative care screening and consultation in the ICU: a multihospital quality improvement project. J Pain Symptom Manage 2017;53:5-12.e3.

20. Tang ST, Liu TW, Lai MS, McCorkle R. Discrepancy in the preferences of place of death between terminally ill cancer patients and their primary family caregivers in Taiwan. Soc Sci Med 2005;61:1560-1566.

21. Parr JD, Zhang B, Nilsson ME, et al. The influence of age on the likelihood of receiving end-of-life care consistent with patient treatment preferences. J Palliat Med 2010;13:719-726.

22. Khan SA, Gomes B, Higginson IJ. End-of-life care-what do cancer patients want? Nat Rev Clin Oncol 2014;11:100-108.

23. Ferrell BR, Temel JS, Temin S, et al. Integration of palliative care into standard oncology care: American Society of Clinical Oncology clinical practice guideline update. J Clin Oncol 2017;35:96-112.

24. Hui D, Kim SH, Roquemore J, et al. Impact of timing and setting of palliative care referral on quality of end-of-life care in cancer patients. Cancer 2014;120:1743-1749.

25. McAdoo SP, Brown EA, Chesser AM, et al. Measuring the quality of end of life management in patients with advanced kidney disease: results from the pan-Thames renal audit group. Nephrol Dial Transplant 2012;27:15481554 . 